\title{
A Multidomain Pseudospectral Mode Solver for Optical Waveguide Analysis
}

\author{
Shun-Fan Chiang, Bang-Yan Lin, Hung-Chun Chang, Senior Member, IEEE, Fellow, OSA, Chun-Hao Teng, \\ Chih-Yu Wang, and Shih-Yung Chung
}

\begin{abstract}
We propose a pseudospectral mode solver for optical waveguide mode analysis formulated by the frequency-domain Maxwell equations. Special attention is paid upon identifying the required boundary operator for the formulation and the relationships between the derived operator and the physical boundary conditions. These theoretical results are adopted into a Legendre pseudospectral multidomain computational framework to compute the propagation characteristics of optical waveguides. Numerical experiments are conducted, and the expected spectral convergence of the scheme is observed for smooth problems and for problems having field jumps at material interfaces. For dielectric waveguides with sharp corners, the spectral convergence is lost due to the singular nature of fields at the corner. Nevertheless, compared with other methods, the present formulation remains as an efficient approach to obtain waveguide modes.
\end{abstract}

Index Terms-Frequency-domain Maxwell's equations, optical waveguides, penalty boundary conditions, pseudospectral methods, waveguide analysis.

\section{INTRODUCTION}

I $\mathrm{N}$ modal analysis for optical waveguides, the propagation constants and the associated field distributions of guided modes provide useful information in designing and operating optical guiding devices such as filters, switches, modulators, and fibers. To obtain these guiding characteristics, one needs to solve either Maxwell's equations or the vectorial Helmholtz

Manuscript received January 19, 2012; revised March 13, 2012; accepted March 17, 2012. Date of publication March 23, 2012; date of current version April 27, 2012. This work was supported in part by the National Science Council of Taiwan under Grant NSC99-2115-M-009-012-MY3, Grant NSC97-2221-E-002-043-MY2, and Grant NSC99-2221-E-002-107-MY2, the Excellent Research Projects of National Taiwan University under Grant 10R80919-1, and the Ministry of Education of Taiwan under The Aim of Top University Plan Grant. The work of C.-H. Teng was supported by the National Science Council of Taiwan under Research Fellow Program NSC099-2811-M-009-047 and NSC100-2811-M-009-055.

S.-F. Chiang was with the Graduate Institute of Photonics and Optoelectronics Engineering, National Taiwan University, Taipei 10617, Taiwan. He is now with Hon Hai Precision Industry Company Ltd., Taipei 20306, Taiwan (e-mail: r95941007@ntu.edu.tw).

B.-Y. Lin was with the Graduate Institute of Communication Engineering, National Taiwan University, Taipei 10617, Taiwan. He is now with Taiwan Semiconductor Manufacturing Company, Hsinchu 30077, Taiwan (e-mail: bangyan.lin@gmail.com).

H.-C. Chang is with the Department of Electric Engineering, National Taiwan University, Taipei 10617, Taiwan (e-mail: hcchang@cc.ee.ntu.edu.tw).

C.-H. Teng is with the Department of Applied Mathematics and the Center of Mathematical Modeling, National Chiao Tung University, Hsinchu 30010, Taiwan (e-mail: tengch@math.nctu.edu.tw).

C.-Y. Wang and S.-Y. Chung are with the Graduate Institute of Electronics Engineering, National Taiwan University, Taipei 10617, Taiwan (e-mail: d95943034@ntu.edu.tw; d95943006@ntu.edu.tw).

Digital Object Identifier 10.1109/JLT.2012.2191937 equations, subject to boundary conditions. However, due to the involved mathematical difficulties raised from either the geometry configurations of devices or the heterogeneous distributions of material properties, waveguide problems are in general very hard to solve analytically. For realistic cases, numerical methods are employed to obtain the guiding characteristics.

Among numerical methods, Cartesian finite difference methods [1]-[6] are popular for waveguide problems if the geometry of the guiding structure is confined to the grid lines. However, for problems involving curved interfaces, special difference stencils are required to treat field values in the vicinity of curved interfaces to maintain accurate computations [7]-[9]. Also commonly used in modal analysis, body-fitted finite element methods [10]-[13] adopt unstructured and structural meshes to fit the geometries and employ edge elements and tangential elements to discretize the equations and boundary conditions. Most of the aforementioned methods are low-order accurate methods, typically first or second order. As the complexity of waveguide problems increases, these finite difference and finite element schemes require using dense meshes to perform accurate computations. Hence, these approaches lead to large systems of equations to be solved, which may consume lots of computational resources and time. An approach for overcoming this issue is designing high-order accurate schemes based on spectral/pseudospectral methods [14]-[17]. Generally, these schemes can compute accurate results by using a coarse mesh, compared to low-order methods. However, this advantage does not come along freely, and great care must be exercised to ensure the high-order convergence rate, because these methods are very sensitive to the smoothness of the solutions and the imposition of boundary conditions [18].

In this study, we present a high-order accurate and geometrically flexible computational approach for waveguide mode analysis. Unlike the mentioned pseudospectral approaches that adopt the vectorial Helmholtz equations as the main equations, we consider the frequency-domain Maxwell's equations as the governing equations. Special attention is paid upon analyzing the required boundary operator for the frequency-domain Maxwell's equations and its relationships with the common physical boundary conditions. These analytic results are then adopted into a multidomain pseudospectral computational framework through the penalty methodology [19]-[22]. The proposed formulation is validated through computing the propagation characteristics of fundamental modes of a number of waveguide structures. We observe the expected spectral convergence results for smooth waveguide problems and for problems having finite jumps of fields at material interfaces. However, for dielectric waveguides having sharp corners, it is found that the spectral convergence is lost, due to the singular 
nature of optical fields at the corner. Nevertheless, the results show that the present scheme remains competitive compared to other finite difference approaches on solving the same problem. Preliminary results of this study were reported in [23]. In this paper, we give the detailed formulation with numerous numerical examples.

The rest of this paper is organized as follows. In Section II, we present the mathematical formulation of the concerned problems and seek the required boundary operator as well as its relationships with common physical boundary conditions to complete the formulation. Section III is devoted to the construction of the discrete scheme of the formulation based on the multidomain pseudospectral penalty approach. The validation of the proposed formulation is presented in Section IV. Concluding remarks are given in Section V.

\section{MATHEMATICAL FORMULATION}

\section{A. Maxwell's Equations}

The Maxwell equations for optical fields in linear, lossless, source-free, and nonmagnetic medium are

$$
\begin{aligned}
\varepsilon_{r} c^{-1} \partial_{t} \hat{E} & =Z_{0} \nabla \times \hat{H} \\
-Z_{0} c^{-1} \partial_{t} \hat{H} & =\nabla \times \hat{E} \\
\nabla \cdot \hat{H} & =0 \\
\nabla \cdot \hat{E} & =0
\end{aligned}
$$

where $\varepsilon_{0}, \mu_{0}, Z_{0}=\left(\mu_{0} / \varepsilon_{0}\right)^{1 / 2}$, and $c=1 /\left(\varepsilon_{0} \mu_{0}\right)^{1 / 2}$ are the permittivity, permeability, impedance, and speed of light in free space, respectively, $\varepsilon_{r}$ assumed real constant is the relative permittivity of medium, $\hat{E}$ and $\hat{H}$ are the electric and magnetic fields, respectively, and $\partial_{t}$ denotes the partial differential operator with respective to $t$.

Let the $z$ axis be the propagation direction. To solve the transverse wave fields in waveguides, we assume the fields having the space-time dependence of the form

$$
\left(\hat{E}, Z_{0} \hat{H}\right)(x, y, z, t)=(E, H)(x, y) \exp (i \beta z-i \omega t)
$$

where $i=\sqrt{-1}, E$ and $H$ are the normalized complex-valued phasors of $\hat{E}$ and $Z_{0} \hat{H}$, respectively, $\beta$ is the propagation constant, and $\omega$ is the angular frequency. Substitution of (2) into (1) leads to the phasor component equations

$$
\begin{aligned}
i \beta E_{x} & =i k_{0} H_{y}+\partial_{x} E_{z} \\
i \beta E_{y} & =-i k_{0} H_{x}+\partial_{y} E_{z} \\
i \beta E_{z} & =-\partial_{x} E_{x}-\partial_{y} E_{y} \\
i \beta H_{x} & =-i k_{0} \varepsilon_{r} E_{y}+\partial_{x} H_{z} \\
i \beta H_{y} & =i k_{0} \varepsilon_{r} E_{x}+\partial_{y} H_{z} \\
i \beta H_{z} & =-\partial_{x} H_{x}-\partial_{y} H_{y} \\
\partial_{x} E_{y} & =i k_{0} H_{z}+\partial_{y} E_{x} \\
\partial_{x} H_{y} & =-i k_{0} \varepsilon_{r} E_{z}+\partial_{y} H_{x}
\end{aligned}
$$

where $k_{0}=\omega / c$ is the space wavenumber in vacuum. Notice that (3g)-(3h) can be derived from (3a)-(3e). We have six independent equations governing the six field variables formulated as an eigensystem, with $\beta$ and the phasor fields being the eigenvalue and eigenvectors, respectively.

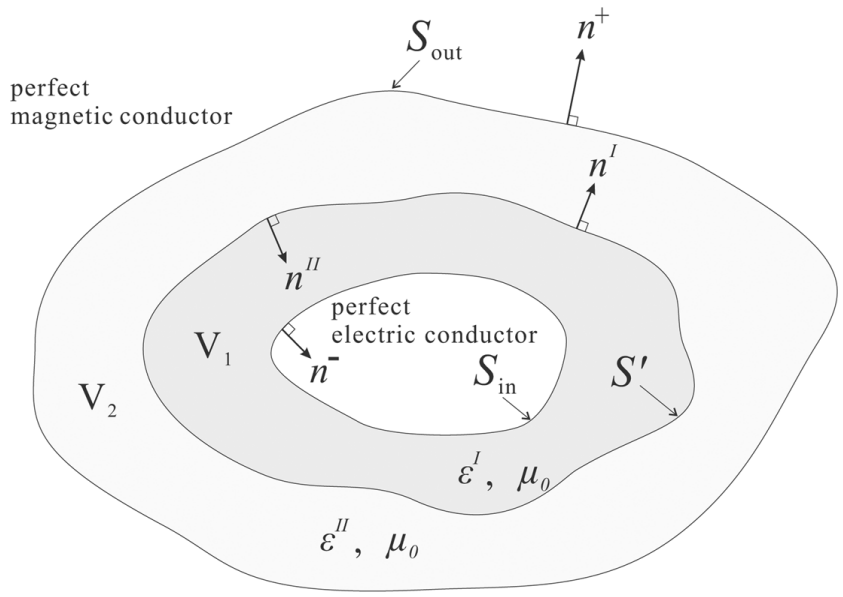

Fig. 1. Schematic view of the cross section of a waveguide.

\section{B. Boundary Operators}

Consider the waveguide having a cross section as depicted in Fig. 1. The structure is closed by perfect magnetic conductor (PMC) and perfect electric conductor (PEC) at the outer and inner surfaces, $S_{\text {out }}$ and $S_{\text {in }}$, respectively. Within the structure, the surface $S^{\prime}$ divides the guiding area into two regions $V_{1}$ and $V_{2}$, characterized by the permittivity and permeability of the material, $\left(\varepsilon^{I}, \mu_{0}\right)$ and $\left(\varepsilon^{I I}, \mu_{0}\right)$, respectively. $n^{-}$and $n^{+}$denote the outward pointing unit vectors normal to the surfaces $S_{\text {in }}$ and $S_{\text {out }}$, respectively, and $n^{I}$ and $n^{I I}$ are unit vectors normal to the surface $S^{\prime}$ pointing from region $V_{1}$ to region $V_{2}$ and from region $V_{2}$ to region $V_{1}$, respectively.

To seek the boundary operator required by (3a)-(3e), we rewrite these equations in the matrix form

$$
i \beta A_{3} q=-i k_{0} A_{0} q+A_{1} \partial_{x} q+A_{2} \partial_{y} q
$$

where $q=\left[\varepsilon_{r} E_{x}, \varepsilon_{r} E_{y}, E_{z}, H_{x}, H_{y}, H_{z}\right]^{T}$ is the state vector with $T$ denoting the vector transpose, and for $i=0,1,2,3$

$$
A_{i}=\left[\begin{array}{cccccc}
\delta_{3 i} \varepsilon_{r}^{-1} & 0 & \delta_{1 i} & 0 & -\delta_{0 i} & 0 \\
0 & \delta_{3 i} \varepsilon_{r}^{-1} & \delta_{i 2} & \delta_{0 i} & 0 & 0 \\
\delta_{1 i} & \delta_{i 2} & -\delta_{3 i} \varepsilon_{r} & 0 & 0 & 0 \\
0 & \delta_{0 i} & 0 & \delta_{3 i} & 0 & \delta_{1 i} \\
-\delta_{0 i} & 0 & 0 & 0 & \delta_{3 i} & \delta_{i 2} \\
0 & 0 & 0 & \delta_{1 i} & \delta_{i 2} & -\delta_{3 i}
\end{array}\right]
$$

with $\delta_{i j}$ being the Kronecker delta function. Multiplying (4) by $q^{*}$ from the left, where $(\cdot)^{*}$ denotes the Hermitian of the variable $(\cdot)$, and multiplying the Hermitian of (4) by $q$ from the right, integrating the entire domain $V_{1} \cup V_{2}$, summing the resultants, and invoking the divergence theorem to change the volume integrals to surface integrals, we obtain

$$
\begin{array}{rl}
0=\int_{S_{\mathrm{in}}} q^{*} & A\left(n^{-}\right) q d x+\int_{S_{I}^{\prime}} q^{*} A\left(n^{I}\right) q d x \\
& +\int_{S_{I I}^{\prime}} q^{*} A\left(n^{I I}\right) q d x+\int_{S_{\mathrm{out}}} q^{*} A\left(n^{+}\right) q d x
\end{array}
$$

where $A(n)=n_{x} A_{1}+n_{y} A_{2}$ with $n=\left(n_{x}, n_{y}\right)$ being the outward pointing unit vector normal to a domain boundary, and $\int_{S}(\cdot) d x$ denotes the integration along the path $S$. Note that the second and the third integrations on the right-hand side (RHS) 
of (5) are evaluated with variables defined on the interface $S^{\prime}$ but in regions $V_{1}$ and $V_{2}$, respectively.

To proceed further, we introduce the characteristic variables at the boundaries. Since $A(n)=A^{T}(n)$, there exists a unitary matrix $S(n)$ such that

$$
S^{*} A(n) S=\Lambda=\operatorname{diag}\left(\lambda_{1}, \lambda_{2}, \lambda_{3}, \lambda_{4}, \lambda_{5}, \lambda_{6}\right)
$$

with $\lambda_{1}=\lambda_{6}=-1, \lambda_{2}=\lambda_{5}=1$, and $\lambda_{3}=\lambda_{4}=0$. Notice that $S^{*}=S^{T}=S^{-1}$ because $A(n)$ is real. Employing $S$, we define the characteristic state vector

$$
R=\left[R_{1}, R_{2}, R_{3}, R_{4}, R_{5}, R_{6}\right]^{T}=S^{*} q
$$

The explicit expressions of $S$ and $R$ are

$$
\begin{aligned}
& S(n)=\frac{1}{\sqrt{2}}\left[\begin{array}{cccccc}
n_{x} & n_{x} & \sqrt{2} n_{y} & 0 & 0 & 0 \\
n_{y} & n_{y} & -\sqrt{2} n_{x} & 0 & 0 & 0 \\
-1 & 1 & 0 & 0 & 0 & 0 \\
0 & 0 & 0 & \sqrt{2} n_{y} & n_{x} & n_{x} \\
0 & 0 & 0 & -\sqrt{2} n_{x} & n_{y} & n_{y} \\
0 & 0 & 0 & 0 & 1 & -1
\end{array}\right] \\
& R(n)=\frac{1}{\sqrt{2}}\left[\begin{array}{c}
\varepsilon_{r}\left(n \cdot E_{\|}\right)-e_{z} \cdot E_{\perp} \\
\varepsilon_{r}\left(n \cdot E_{\|}\right)+e_{z} \cdot E_{\perp} \\
-\sqrt{2} \varepsilon_{r} e_{z} \cdot\left(n \times E_{\|}\right) \\
-\sqrt{2} e_{z} \cdot\left(n \times H_{\|}\right) \\
\left(n \cdot H_{\|}\right)+e_{z} \cdot H_{\perp} \\
\left(n \cdot H_{\|}\right)-e_{z} \cdot H_{\perp}
\end{array}\right]
\end{aligned}
$$

where $e_{z}$ is the unit vector in the $z$-direction, $E_{\|}\left(H_{\|}\right)$is the transverse electric (magnetic) field, and $E_{\perp}\left(H_{\perp}\right)$ is the electric (magnetic) field perpendicular to the $x y$ plane.

Equation (5) implies that the required boundary conditions must lead to the vanishing of the RHS of the equation. We now examine whether the specified boundary conditions satisfy this constrain.

a) PMC Condition at $S_{\text {out }}$ : The conditions are

$$
n^{+} \cdot E_{\|}=0, \quad n^{+} \times H_{\|}=0, \quad e_{z} \cdot H_{\perp}=0 .
$$

It follows from (7), $n^{+} \cdot E_{\|}=0$ and $e_{z} \cdot H_{\perp}=0$, that

$$
\begin{aligned}
& R_{1}\left(n^{+}\right)+R_{2}\left(n^{+}\right)=\sqrt{2} n^{+} \cdot \varepsilon_{r} E_{\|}=0 \\
& R_{5}\left(n^{+}\right)-R_{6}\left(n^{+}\right)=\sqrt{2} e_{z} \cdot H_{\perp}=0 .
\end{aligned}
$$

Thus, $\left|R_{1}\left(n^{+}\right)\right|^{2}=\left|R_{2}\left(n^{+}\right)\right|^{2}$ and $\left|R_{5}\left(n^{+}\right)\right|^{2}=\left|R_{6}\left(n^{+}\right)\right|^{2}$. Furthermore, since $\lambda_{3}=\lambda_{4}=0$, we obtain

$$
q^{*} A\left(n^{+}\right) q=R^{*}\left(n^{+}\right) \Lambda R\left(n^{+}\right)=0
$$

indicating that $\int_{S_{\text {out }}} q^{*} A\left(n^{+}\right) q d x=0$ in (5).

b) PEC at $S_{\mathrm{in}}^{\text {: }}$ : The conditions are

$$
n^{-} \times E_{\|}=0, \quad e_{z} \cdot E_{\perp}=0, \quad n^{-} \cdot H_{\|}=0 .
$$

It follows from (7), $e_{z} \cdot E_{\perp}=0$ and $n^{-} \cdot H_{\|}=0$, that

$$
\begin{aligned}
& R_{1}\left(n^{-}\right)-R_{2}\left(n^{-}\right)=-\sqrt{2} e_{z} \cdot E_{\perp}=0 \\
& R_{5}\left(n^{-}\right)+R_{6}\left(n^{-}\right)=\sqrt{2} n^{-} \cdot H=0 .
\end{aligned}
$$

Thus, $\left|R_{1}\left(n^{-}\right)\right|=\left|R_{2}\left(n^{-}\right)\right|^{2}$ and $\left|R_{5}\left(n^{-}\right)\right|^{2}=\left|R_{6}\left(n^{-}\right)\right|^{2}$. Since $\lambda_{3}=\lambda_{4}=0$, we obtain

$$
q^{*} A\left(n^{-}\right) q=R^{*}\left(n^{-}\right) \Lambda R\left(n^{-}\right)=0
$$

indicating that $\int_{S_{\text {in }}} q^{*} A\left(n^{-}\right) q d x=0$ in (5).

c) Material Interface Condition at $S^{\prime}$ : The interface boundary conditions relating fields on both sides of the interface $S^{\prime}$ are

$$
\begin{aligned}
n^{I} \times E_{\|}^{I} & =n^{I} \times E_{\|}^{I I} \\
e_{z} \cdot E_{\perp}^{I} & =e_{z} \cdot E_{\perp}^{I I} \\
n^{I} \cdot \varepsilon^{I} E_{\|}^{I} & =n^{I} \cdot \varepsilon^{I I} E_{\|}^{I I} \\
n^{I} \times H_{\|}^{I} & =n^{I} \times H_{\|}^{I I} \\
e_{z} \cdot H_{\perp}^{I} & =e_{z} \cdot H_{\perp}^{I I} \\
n^{I} \cdot H_{\|}^{I} & =n^{I} \cdot H_{\|}^{I I}
\end{aligned}
$$

where $n^{I}$ is the unit vector normal to $S^{\prime}$ pointing from region $V_{1}$ to region $V_{2}$, and the superscripts $I$ and $I I$ denote the regions where the field variables are defined on the $S^{\prime}$ interface, respectively. From (7), we have

$$
\begin{aligned}
\sqrt{2} R_{1}^{I}\left(n^{I}\right) & =\varepsilon_{r}^{I} n^{I} \cdot E_{\|}^{I}-e_{z} \cdot E_{\perp}^{I} \\
\sqrt{2} R_{2}^{I I}\left(n^{I I}\right) & =\varepsilon_{r}^{I I} n^{I I} \cdot E_{\|}^{I I}+e_{z} \cdot E_{\perp}^{I I} .
\end{aligned}
$$

Invoking (10b) and (10c) as well as $n^{I}=-n^{I I}$, we yield

$$
R_{1}^{I}\left(n^{I}\right)=-R_{2}^{I I}\left(n^{I I}\right) .
$$

Following similar procedures, we obtain the conditions

$$
\begin{aligned}
& R_{2}^{I}\left(n^{I}\right)=-R_{1}^{I I}\left(n^{I I}\right) \\
& R_{5}^{I}\left(n^{I}\right)=-R_{6}^{I I}\left(n^{I I}\right) \\
& R_{6}^{I}\left(n^{I}\right)=-R_{5}^{I I}\left(n^{I I}\right) .
\end{aligned}
$$

It follows from these expressions and $\lambda_{3}=\lambda_{4}=0$ that

$$
\begin{aligned}
q^{*} A\left(n^{I}\right) q & =R^{*}\left(n^{I}\right) \Lambda R\left(n^{I}\right) \\
& =-R^{*}\left(n^{I I}\right) \Lambda R\left(n^{I I}\right) \\
& =-q^{*} A\left(n^{I I}\right) q .
\end{aligned}
$$

Therefore, $\int_{S_{I}^{\prime}} q^{*} A\left(n^{-}\right) q d x+\int_{S^{\prime}{ }^{\prime}} q^{*} A\left(n^{+}\right) q\left(n^{+}\right) d x=0$.

The analysis shows that to satisfy (5), we need to specify $R_{1}$, $R_{2}, R_{5}$, and $R_{6}$ on the boundary to complete the system. The explicit expressions of these characteristic components and their relationships with the common physical boundary conditions are established. In the next section, we shall apply these result and construct a pseudospectral penalty scheme for solving wave fields in waveguides.

\section{NUMERICAL FORMULATION}

\section{A. Legendre Pseudospectral Method}

Let I $=[-1,1]$ and $N$ be a positive integer. Introduce the Legendre-Gauss-Lobatto (LGL) points $\xi_{i}$ for $0 \leq i \leq N$. These points are roots of the polynomial $\left(1-\xi^{2}\right) P_{N}^{\prime}(\xi)$ where $P_{N}(\xi)$ is the $N$ th degree Legendre polynomial and 'denotes 

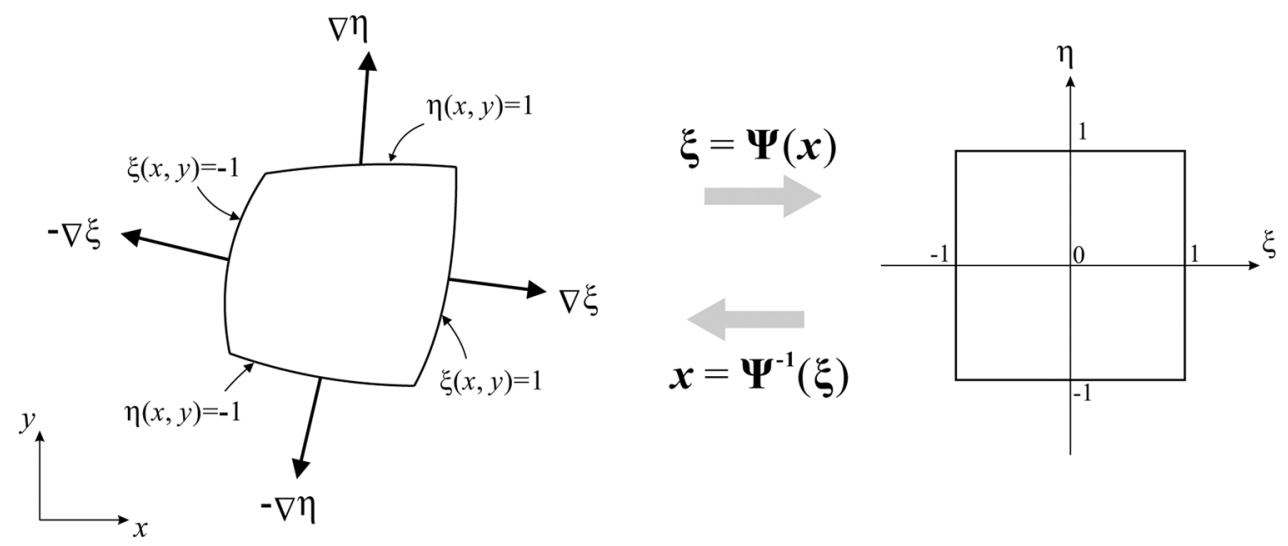

Fig. 2. Coordinate mapping of a square in $\xi=(\xi, \eta)$ coordinate and a general curvilinear quadrilateral in $\boldsymbol{x}=(x, y)$ coordinate.

the differentiation. To approximate a function $f$ defined on I, we seek a polynomial $f_{N}$ of the form

$$
\begin{aligned}
f_{N}(\xi) & =\sum_{j=0}^{N} l_{j}(\xi) f_{j} \\
l_{j}(\xi) & =-\frac{\left(1-\xi^{2}\right) P_{N}^{\prime}(\xi)}{N(N+1)\left(\xi-\xi_{j}\right) P_{N}\left(\xi_{j}\right)}
\end{aligned}
$$

where $f_{j}=f\left(\xi_{j}\right)$ and $l_{j}(\xi)$ are the Lagrange interpolating functions. The 1-D pseudospectral method can be extended to a 2-D framework through the tensor product formulation. Let $N_{\xi}$ and $N_{\eta}$ be positive integers. Denote $\left.\right|^{2}=[-1,1]^{2}$ and term the coordinates $(\xi, \eta)$. Consider the grid points $\xi_{i}$ for $0 \leq i \leq N_{\xi}$ and $\eta_{j}$ for $0 \leq j \leq N_{\eta}$, where $\xi_{i}$ and $\eta_{j}$ are the LGL grid points along the $\xi$ - and $\eta$-axis, respectively. Then, the grid points are defined as $\left(\xi_{i}, \eta_{j}\right)$. To approximate $f(\xi, \eta)$ defined on $\mathrm{I}^{2}$, we construct a polynomial $f_{N}(\xi, \eta)$ of the form

$$
f_{N}(\xi, \eta)=\sum_{j=0}^{N_{\eta}} \sum_{i=0}^{N_{\xi}} l_{i}^{\xi}(\xi) l_{j}^{\eta}(\eta) f_{i j}, \quad f_{i j}=f\left(\xi_{i}, \eta_{j}\right)
$$

where $l_{i}^{\xi}(\xi)$ and $l_{j}^{\eta}(\eta)$ are the 1-D Lagrange interpolating functions based on the grid points $\xi_{i}$ and $\eta_{j}$, respectively. The partial derivatives of $f$ are approximated as

$$
\begin{aligned}
& \partial_{\xi} f\left(\xi_{p}, \eta_{q}\right) \approx \partial_{\xi} f_{N}\left(\xi_{p}, \eta_{q}\right)=\sum_{j=0}^{N_{\eta}} \sum_{i=0}^{N_{\xi}} \frac{d l_{i}^{\xi}\left(\xi_{p}\right)}{d \xi} l_{j}^{\eta}\left(\eta_{q}\right) f_{i j} \\
& \partial_{\eta} f\left(\xi_{p}, \eta_{q}\right) \approx \partial_{\eta} f_{N}\left(\xi_{p}, \eta_{q}\right)=\sum_{j=0}^{N_{\eta}} \sum_{i=0}^{N_{\xi}} l_{i}^{\xi}\left(\xi_{p}\right) \frac{d l_{j}^{\eta}\left(\eta_{q}\right)}{d \eta} f_{i j} .
\end{aligned}
$$

We have reviewed some basic concepts related to the Legendre pseudospectral method. For further details on the method, we refer the reader to [18].

\section{B. Equations in Curvilinear Coordinates}

The tensor-product-based pseudospectral formulation is for problems defined on standard domain $[-1,1]^{2}$. To apply the formulation for problems defined on general complex domains, an approach is decomposing the global domains into unions of smooth quadrilateral subdomains which can be mapped onto a standard domain. We understand that establishing a general coordinate transformation for mapping an arbitrary quadrilateral domain onto a square domain may not be always possible. Hence, we describe the methodology for problems defined on a curvilinear quadrilateral element that can be mapped onto a square.

Consider a general curvilinear quadrilateral $\Omega$ and a square $1^{2}$ defined in coordinate systems termed $(x, y)$ and $(\xi, \eta)$, respectively (see Fig. 2). We apply the transfinite blending interpolation method [24] and construct a coordinate mapping $(\xi, \eta)=\Psi(x, y)$ and its inverse $(x, y)=\Psi^{-1}(\xi, \eta)$ to establish a one-to-one correspondence between $\Omega$ and $I^{2}$. The transformation metric functions are then computed as

$$
\frac{\partial(\xi, \eta)}{\partial(x, y)}=\left[\begin{array}{cc}
\partial_{x} \xi & \partial_{y} \xi \\
\partial_{x} \eta & \partial_{y} \eta
\end{array}\right], \quad \frac{\partial(x, y)}{\partial(\xi, \eta)}=\left[\begin{array}{cc}
\partial_{\xi} x & \partial_{\eta} x \\
\partial_{\xi} y & \partial_{\eta} y
\end{array}\right]
$$

and they are related as $(\partial(\xi, \eta)) /(\partial(x, y))(\partial(x, y)) /$ $(\partial(\xi, \eta))=I$ where $I$ is the identity matrix. Using the coordinate transformation and the chain rule of differentiation, we rewrite (4) as

$$
i \beta \mathrm{A}_{z} \mathrm{q}=-i \omega \mathrm{A}_{t} \mathrm{q}+\mathrm{A}_{\xi} \partial_{\xi} \mathrm{q}+\mathrm{A}_{\eta} \partial_{\eta} \mathrm{q}
$$

where $\mathrm{q}(\xi, \eta)=q\left(\Psi^{-1}(\xi, \eta)\right), \mathrm{A}_{3}=A_{3}, \mathrm{~A}_{0}=A_{0}, \mathrm{~A}_{\xi}=$ $A_{1} \partial_{x} \xi+A_{2} \partial_{y} \xi$, and $\mathrm{A}_{\eta}=A_{1} \partial_{x} \eta+A_{2} \partial_{y} \eta$.

Denote $\mathrm{n}=\left(\mathrm{n}_{\xi}, \mathrm{n}_{\eta}\right)$ as the unit vector normal to the boundary of $\mathrm{I}^{2}$ in the $(\xi, \eta)$ coordinate system. On the boundary of $\mathrm{I}^{2}$, we define the variables $A(n), S(n), \Gamma(n)$ and $R(n)$, related by $A(n)=n_{\xi} A_{\xi}+n_{\eta} A_{\eta}, S^{*}(n) A(n) S(n)=\Gamma(n)$, and $R=$ $S^{*}(n) q$. The variables $S, \Gamma$, and $R$ and their transformed correspondences $S, \Lambda$, and $R$ defined on the boundary of $\Omega$ are related as follows. Denote the unit vectors along the $\xi$ - and $\eta$-axes by $\hat{\xi}=(1,0)$ and $\hat{\eta}=(0,1)$, respectively. On the edges $\xi(x, y)=$ \pm 1 , we have $\Gamma( \pm \hat{\xi})=|\nabla \xi| \Lambda, \mathrm{S}( \pm \hat{\xi})=S( \pm \nabla \xi /|\nabla \xi|)$, and $\mathrm{R}( \pm \hat{\xi})=R( \pm \nabla \xi /|\nabla \xi|)$. Replacing the symbol $\xi$ by $\eta$ in the aforementioned expressions, we obtain the corresponding relationships for the variables defined on $\eta(x, y)= \pm 1$. 


\section{Numerical Scheme}

We now present the numerical scheme for solving (15). Let $\mathrm{q}_{i j}$ denote the numerical state vectors at the grid points $\left(\xi_{i}, \eta_{j}\right)$ satisfying the collocation schemes

$$
i \beta \mathrm{A}_{3} \mathrm{q}_{i j}=-i k_{0} \mathrm{~A}_{0} \mathrm{q}_{i j}+\mathrm{A}_{\xi} \partial_{\xi} \mathrm{q}_{i j}+\mathrm{A}_{\eta} \partial_{\eta} \mathrm{q}_{i j}-\frac{1}{2} \sum_{\nu=1}^{4} \mathrm{P}_{i j}^{(\nu)}
$$

where

$$
\begin{aligned}
& \mathrm{P}_{i j}^{(1)}=\delta_{i 0}|\nabla \xi|_{i j}\left(\omega_{i}^{\xi}\right)^{-1} \mathrm{~S}_{i j}(-\hat{\xi}) \Lambda\left(\mathrm{R}_{i j}(-\hat{\xi})-\mathrm{R}_{i j}^{B C}\right) \\
& \mathrm{P}_{i j}^{(2)}=\delta_{i N_{\xi}}|\nabla \xi|_{i j}\left(\omega_{i}^{\xi}\right)^{-1} \mathrm{~S}_{i j}(\hat{\xi}) \Lambda\left(\mathrm{R}_{i j}(\hat{\xi})-\mathrm{R}_{i j}^{B C}\right) \\
& \mathrm{P}_{i j}^{(3)}=\delta_{j 0}|\nabla \eta|_{i j}\left(\omega_{j}^{\eta}\right)^{-1} \mathrm{~S}_{i j}(-\hat{\eta}) \Lambda\left(\mathrm{R}_{i j}(-\hat{\eta})-\mathrm{R}_{i j}^{B C}\right) \\
& \mathrm{P}_{i j}^{(4)}=\delta_{j N_{\eta}}|\nabla \eta|_{i j}\left(\omega_{j}^{\eta}\right)^{-1} \mathrm{~S}_{i j}(\hat{\eta}) \Lambda\left(\mathrm{R}_{i j}(\hat{\eta})-\mathrm{R}_{i j}^{B C}\right)
\end{aligned}
$$

with $\omega_{i}^{\xi}$ and $\omega_{j}^{\eta}$ being the quadrature weights [18] associated with the grid points $\xi_{i}$ and $\eta_{j}$, respectively. It is shown in (16) that the boundary conditions are enforced into the scheme weakly through the characteristic variables.

To complete the construction of the scheme, we need to provide the explicit expression of $\mathrm{R}_{i j}^{B C}$. For simplicity, we consider the imposition of boundary conditions on the $\xi=1$ edge as an example. To impose PMC condition, it is suggested from (8a) and ( $8 \mathrm{~b}$ ) to construct $\mathrm{R}^{B C}=\left[-\mathrm{R}_{2},-\mathrm{R}_{1}, \mathrm{R}_{3}, \mathrm{R}_{4}, \mathrm{R}_{6}, \mathrm{R}_{5}\right]^{T}$, leading to

$$
\begin{aligned}
&|\nabla \xi| \mathrm{S} \Lambda\left(\mathrm{R}-\mathrm{R}^{B C}\right) \\
&=2\left[0,0, \varepsilon_{r} \nabla \xi \cdot \mathrm{E}_{\|},\left(\partial_{x} \xi\right) \mathrm{H}_{z},\left(\partial_{y} \xi\right) \mathrm{H}_{z}, 0\right]^{T}
\end{aligned}
$$

where $\mathrm{E}_{\|}=\left(\mathrm{E}_{x}, \mathrm{E}_{y}\right)$. Likewise, to enforce PEC condition, it is suggested from (9a) and (9b) to construct $\mathrm{R}^{B C}=\left[\mathrm{R}_{2}, \mathrm{R}_{1}, \mathrm{R}_{3}, \mathrm{R}_{4},-\mathrm{R}_{6},-\mathrm{R}_{5}\right]^{T}$. Thus,

$$
|\nabla \xi| \mathrm{S} \Lambda\left(\mathrm{R}-\mathrm{R}^{B C}\right)=2\left[\left(\partial_{x} \xi\right) \mathrm{E}_{z},\left(\partial_{y} \xi\right) \mathrm{E}_{z}, 0,0,0, \nabla \xi \cdot \mathrm{H}_{\|}\right]^{T}
$$

where $\mathrm{H}_{\|}=\left(\mathrm{H}_{x}, \mathrm{H}_{y}\right)$. We now discuss the imposition of interface boundary condition. From (11)-(14), it is suggested to take $\mathrm{R}^{I, B C}=\left[-\mathrm{R}_{2}^{I I},-\mathrm{R}_{1}^{I I}, \mathrm{R}_{3}^{I}, \mathrm{R}_{4}^{I},-\mathrm{R}_{6}^{I I},-\mathrm{R}_{5}^{I I}\right]^{T}$. Hence,

$$
\begin{aligned}
& |\nabla \xi| \mathrm{S} \Lambda\left(\mathrm{R}^{I}-\mathrm{R}^{I, B C}\right) \\
& =\sqrt{2}\left[\left.\left(\partial_{x} \xi\right)\left[\mathrm{E}_{z}\right]\right|_{I I} ^{I},\left.\left(\partial_{y} \xi\right)\left[\mathrm{E}_{z}\right]\right|_{I I} ^{I},\left.\nabla \xi \cdot\left[\varepsilon_{r} \mathrm{E}_{\|}\right]\right|_{I I} ^{I},\right. \\
& \left.\left.\quad\left(\partial_{x} \xi\right)\left[\mathrm{H}_{z}\right]\right|_{I I} ^{I},\left.\left(\partial_{y} \xi\right)\left[\mathrm{H}_{z}\right]\right|_{I I} ^{I},\left.\nabla \xi \cdot\left[\mathrm{H}_{\|}\right]\right|_{I I} ^{I}\right]^{T}
\end{aligned}
$$

where $\left.\left[\mathrm{E}_{z}\right]\right|_{I I} ^{I}=\mathrm{E}_{z}^{I}-\mathrm{E}_{z}^{I I}$ and similarly for the other parallel notations.

By employing (16) and the aforementioned boundary relationship, the wave fields on the transverse plane of a waveguide can be formulated as an eigenvalue problem of the form $\mathcal{A Q}=i \beta \mathcal{Q}$, where the propagation constant $\beta$ is the eigenvalue, $\mathcal{Q}$ is the eigenvector composed of the collocated state vectors, and $\mathcal{A}$ is a matrix. Solving the eigensystem, we obtain $\beta$ and the transverse wave fields.

\section{NUMERICAL VALIDATIONS AND DISCUSSION}

\section{A. Errors of Effective Index and Residual Functions}

We examine the performance of the method by measuring the error of the computed effective index $\left|\delta n_{\text {eff }}\right|$ defined as

$$
\left|\delta n_{\mathrm{eff}}\right|=\left|n_{\mathrm{eff}}-n_{\mathrm{eff}}^{\prime}\right|, \quad n_{\mathrm{eff}}=\beta k_{0}^{-1}=(2 \pi)^{-1} \beta \lambda_{0}
$$

where $n_{\text {eff }}^{\prime}$ and $n_{\text {eff }}$ are the exact and the computed effective indexes and $\lambda_{0}$ is the operating wavelength. To measure how well the computed eigenmode solutions are, we employ the expressions in $(3 \mathrm{~g})$ and $(3 \mathrm{~h})$, which are omitted in computations, and define the residual functions $r_{1}$ and $r_{2}$ as

$$
\begin{aligned}
& r_{1}^{2}=\int_{\Omega}\left|i k_{0} H_{z}+\partial_{y} E_{x}-\partial_{x} E_{y}\right|^{2} d x \\
& r_{2}^{2}=\int_{\Omega}\left|-i k_{0} \varepsilon_{r} E_{z}+\partial_{y} H_{x}-\partial_{x} H_{y}\right|^{2} d x
\end{aligned}
$$

where the integrals are evaluated numerically by the LGL integration quadrature rule [18]. The residual functions can be served as accuracy indicators of the numerical eigenmode solution because if the computed fields are obtained by the scheme convergence to the exact fields of (3a)-(3h), then the residual functions shall decay as the mesh is refined.

The convergence rate of the computed effective index is calculated as

$$
\zeta=\frac{\log \left(\left|\delta n_{\mathrm{eff}}\left(N_{1}\right)\right| /\left|\delta n_{\mathrm{eff}}\left(N_{2}\right)\right|\right)}{\log \left(N_{2} / N_{1}\right)}
$$

where $N_{1}$ and $N_{2}$ are the characterized degrees of the approximation polynomials during mesh refinement. Replacing $\delta n_{\text {eff }}$ in the aforementioned expression by $r_{1}$ and $r_{2}$, we compute the convergence rates of the residual functions $r_{1}$ and $r_{2}$, respectively.

\section{B. Partially Filled Metallic Waveguide}

Consider a half-filled metallic waveguide structure with the geometry and the refractive indexes shown in Fig. 3. The effective index of the fundamental longitudinal-section electric $\left(\mathrm{LSE}_{10}\right)$ mode at the operating wavelength $\lambda_{0}=2 \pi \mu \mathrm{m}$ is solved by the present method with a computational mesh shown in Fig. 3.

A grid convergence study is given in Table I. We see that the error of the effective index decays exponentially as the mesh is refined. The convergence study illustrates the efficiency of the proposed formulation in computing accurate solutions. It is observed that the computed effective index has reached machine accuracy level even using coarse grid meshes. In addition, it is also shown that the residual $r_{1}$ vanishes as the polynomial degree $N$ increases. The values of $r_{2}$ are at machine accuracy level for different values of $N$, because the $E_{z}, H_{x}$, and $H_{y}$ fields are zero for this particular mode.

\section{Circular Metallic Waveguide}

Consider an air-filled circular metallic waveguide with the geometry and the refractive indexes shown in Fig. 4. The effective index for the fundamental transverse electric $\left(\mathrm{TE}_{11}\right)$ mode at the operating wavelength $\lambda_{0}=0.2 \mu \mathrm{m}$ is solved by the 

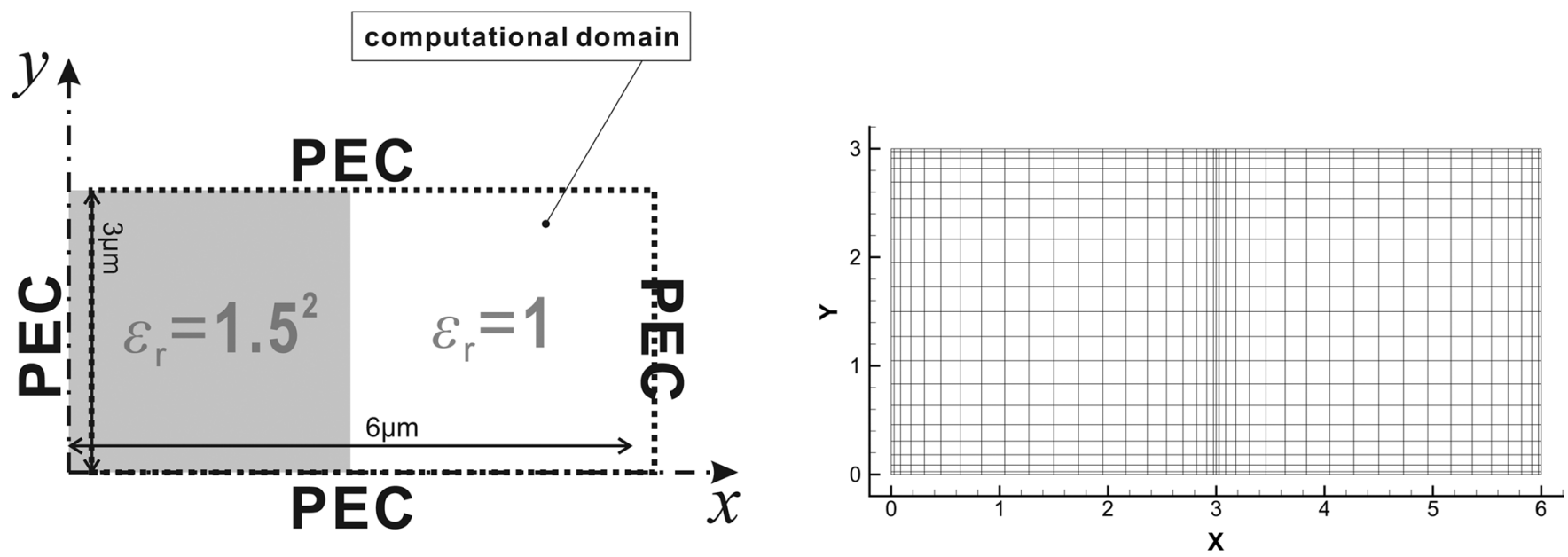

Fig. 3. Schematic view of (left) a partially filled metallic waveguide and (right) a multidomain computational mesh composed of two subdomains. Each subdomain contains $(N+1) \times(N+1)$ grid points, where $N$ is the degree of the approximation polynomial.
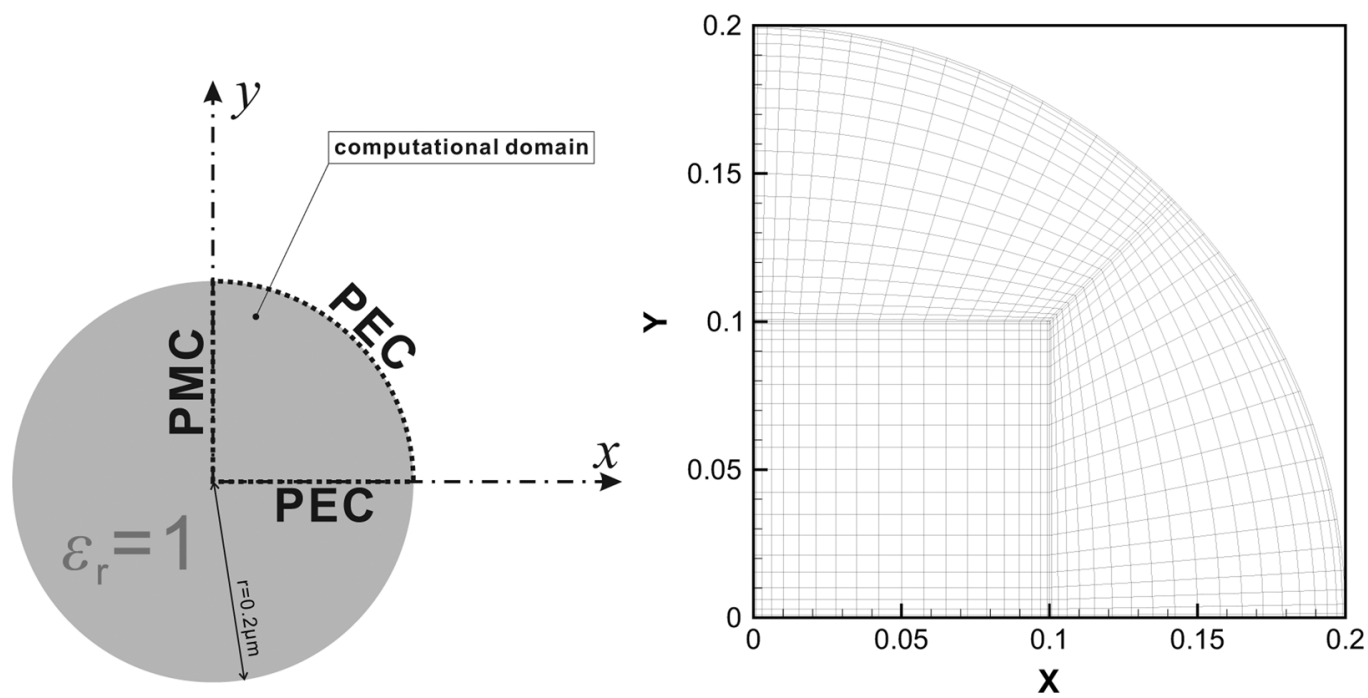

Fig. 4. (Left) Schematic diagram of a circular metallic waveguide. (Right) Multidomain mesh composed of three subdomains.

TABLE I

Convergence Study of the Partially Filled Metallic WaVeguide. $n_{\text {eff }}^{\prime}=1.27575556678727$, REFERENCED EFFECTIVE INDEX FOR THE LSE 10 MOde AT THE OPERATING WAVELENGTH $2 \pi \mu$ M OBTAINED BY SOLVING TRANSCENDENTAL EQUATIONS [25]

\begin{tabular}{||c|c|c||c|c|c|c|c||}
\hline$N$ & $\left|\delta n_{e f f}\right|$ & $\zeta$ & $N$ & $r_{1}$ & $\zeta$ & $r_{2}$ & $\zeta$ \\
\hline 4 & $1.78 \mathrm{E}-04$ & - & 8 & $5.06 \mathrm{E}-05$ & - & $2.84 \mathrm{E}-15$ & - \\
6 & $1.74 \mathrm{E}-08$ & 22.7 & 10 & $2.24 \mathrm{E}-07$ & 24.2 & $4.96 \mathrm{E}-15$ & -2.4 \\
8 & $3.76 \mathrm{E}-12$ & 29.3 & 12 & $1.70 \mathrm{E}-09$ & 26.7 & $3.53 \mathrm{E}-15$ & 1.9 \\
10 & $7.10 \mathrm{E}-15$ & 28.1 & 14 & $4.56 \mathrm{E}-12$ & 38.4 & $3.74 \mathrm{E}-15$ & -0.4 \\
\hline
\end{tabular}

present method. Due to the geometrical symmetry, we only need to solve the problem on the upper right quarter fan region supported by the PEC and PMC conditions at the two straight edges.

A grid convergence study is given in Table II. We observe that the error of the computed effective index and the residuals $r_{1}$ and $r_{2}$ vanish rapidly to machine accuracy level, as $N$ increases. The results indicate that the present formulations incorporated with the transfinite blending mapping also perform very well for solving problems involving curved boundary.
TABLE II

Convergence Study of the Circular Metallic Waveguide. $n_{\text {eff }}^{\prime}=0.9561021744104193$, REFERENCED EFFECTIVE INDEX FOR THE $\mathrm{TE}_{11}$ Mode at the Operating Wavelength $\lambda_{0}=0.2 \mu \mathrm{M}$

\begin{tabular}{||c|c|c||c|c|c|c|c||}
\hline$N$ & $\left|\delta n_{e f f}\right|$ & $\zeta$ & $N$ & $r_{1}$ & $\zeta$ & $r_{2}$ & $\zeta$ \\
\hline 3 & $1.49 \mathrm{E}-06$ & - & 6 & $2.24 \mathrm{E}-06$ & - & $1.20 \mathrm{E}-06$ & - \\
4 & $4.30 \mathrm{E}-08$ & 12.3 & 8 & $2.68 \mathrm{E}-08$ & 15.3 & $1.60 \mathrm{E}-08$ & 15.0 \\
5 & $1.47 \mathrm{E}-10$ & 25.4 & 10 & $4.95 \mathrm{E}-11$ & 28.2 & $2.53 \mathrm{E}-11$ & 28.9 \\
6 & $2.42 \mathrm{E}-12$ & 22.5 & 12 & $1.91 \mathrm{E}-12$ & 17.8 & $1.25 \mathrm{E}-12$ & 16.5 \\
7 & $1.02 \mathrm{E}-14$ & 35.4 & 14 & $1.02 \mathrm{E}-12$ & 4.06 & $7.81 \mathrm{E}-13$ & 3.04 \\
\hline
\end{tabular}

\section{Fiber Waveguide}

Consider a fiber waveguide with the geometry and the refractive indicies as shown in Fig. 5. We use the present method to solve the effective index for the fundamental $\mathrm{HE}_{11}$ mode of the fiber waveguide at the operating wavelength $\lambda_{0}=1.5 \mu \mathrm{m}$. Notice that this problem has a high index difference between the core and the surrounding areas, which causes fields having jumps at the dielectric interface. Thus, this problem is suitable 


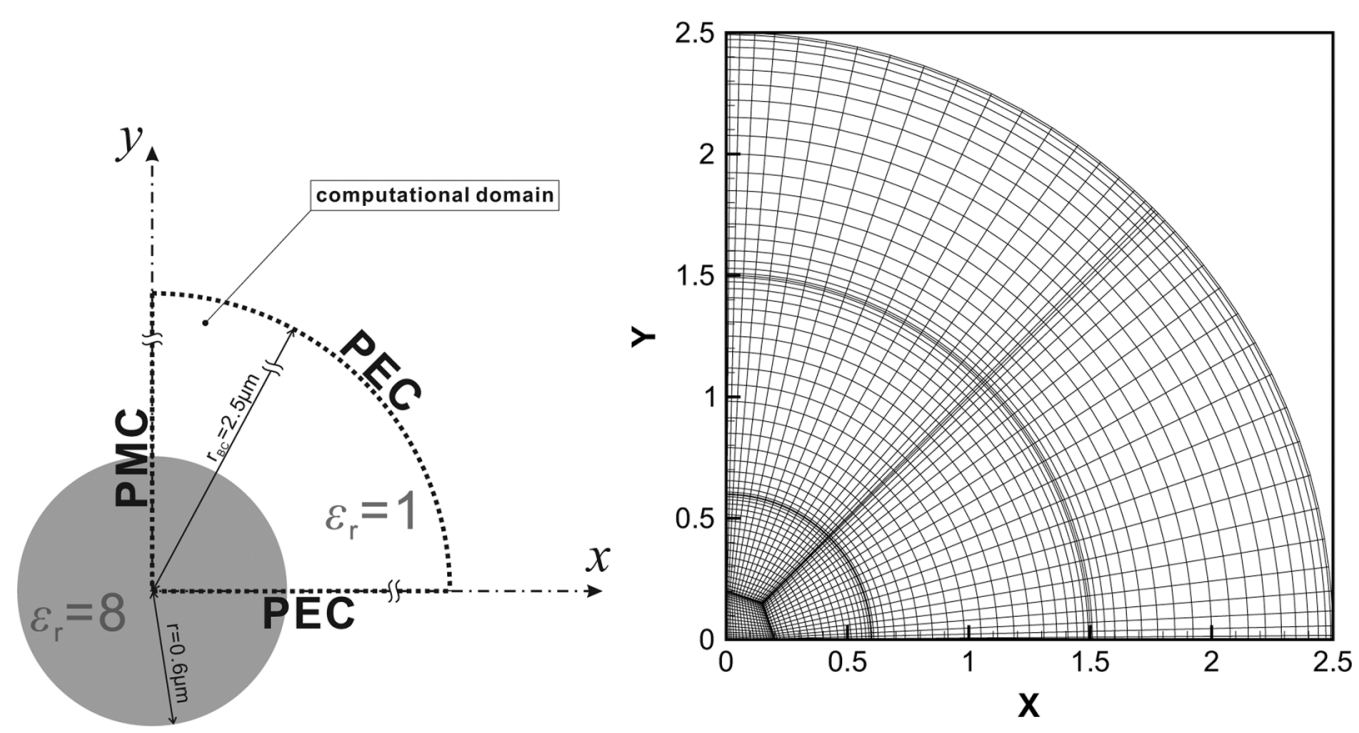

Fig. 5. Schematic view of (left) a fiber waveguide and (right) a multidomain mesh.

for validating the numerical boundary impositions of the present formulation.

Notice that the problem domain radially extends to infinity, which cannot be directly solved by the present formulation for problems defined on finite regions. However, since the fields in the air region are of evanescent type, the amplitude of each field vanishes in the far zone. Employing this property, we terminate the domain by a virtual boundary and enforce PEC or PMC condition at the artificial closure. As will be shown soon, such an arrangement does not harm the computation accuracy provided that the artificial boundary is far away from the fiber core. Furthermore, since the problem domain possesses circular symmetry, it is sufficient enough to consider the problem defined on the upper right quarter of the waveguide cross section. We use a mesh composed of seven subdomains as shown in Fig. 5.

A grid convergence study is provided in Table III. It is shown that the errors of the computed effective index and the residual functions $r_{1}$ and $r_{2}$ all vanish exponentially as the mesh is refined. We also obtain $n_{\text {eff }}=2.6840193216091506$ when using $N=15$, which gives a $\left|\delta n_{\text {eff }}\right|$ less than $10^{-14}$. The computed fields of the fundamental $\mathrm{HE}_{11}$ mode with $N=20$ are shown in Fig. 6. We observe that the field components are mostly confined in the guiding core area and in the vicinity of the dielectric interface, and the amplitude of each field component decays to zero away from the core area. We observe the field jumps at the circular dielectric interface, indicating that the proposed boundary formulation does well capture the essential character of fields at the material interface.

\section{E. Channel Waveguide With Sharp Corners}

We now examine the performance of the formulation on solving propagation characteristics of a square channel waveguide with the geometry and the refractive indexes shown in Fig. 7. A special feature of the considered dielectric channel waveguide is that the electric fields at the dielectric sharp corners are singular (see [5] and the references therein). There, an analysis was conducted to compute the propagation characteristics involving the singular behavior of fields at sharp corners and the result obtained by the difference method is adopted here as a reference.

A grid convergence study is provided in Table IV. We observe that the residual functions $r_{1}$ and $r_{2}$ only decay in three-quarter rate at most and a second-order rate, respectively, as the computational mesh is refined. The spectral converge rate is ruined due to the weakly divergent behavior of the $E_{x}$ and $E_{y}$ field components at the sharp corner [5], [13], which indeed has a great impact on the convergence of the scheme. In our computation, it is found that the local residual $\left|i k_{0} H_{z}+\partial E_{x} / \partial y-\partial E_{y} / \partial x\right|^{2}$ at the corner does not decay. This ill behavior, thus, leads to a very poor convergence of the residual $r_{1}$. On the other hand, it is found that the local residual $\left|-i k_{0} \varepsilon_{r} E_{z}+\partial H_{x} / \partial y-\partial H_{y} / \partial x\right|$ at the corner decays in the first-order rate. As a consequence, the residual function $r_{2}$ vanishes in a second-order rate.

Although the spectral convergence of the scheme is lost for this problem, it is shown that the effective index obtained by the present method still agrees well with the referenced one. In fact, from the computed results, we observe that by employing a coarse grid mesh (four subdomains with $7 \times 7$ grid points in each subdomain), it is sufficient enough to compute the effective index having a similar accuracy as the referenced one obtained by a $150 \times 150$ finite difference grid mesh. This advantage allows one to compute more accurate results by increasing the resolution of the grid mesh. We close this section by providing more accurate results of the effective index of the channel waveguide for $\varepsilon_{r}=2.25$ in Table $\mathrm{V}$ and for $\varepsilon_{r}=8$ in Table VI. It is shown that the effective indexes are approximately 1.276274037 for $\varepsilon_{r}=2.25$ and approximately 2.656796923 for $\varepsilon_{r}=8$. These values are consistent with those obtained by a recently developed waveguide mode solver based on Neumann-to-Dirichlet operators and boundary integral equations [26], in which an accuracy comparison has been made with the result in [23].

\section{CONCLUding Remarks}

We have presented a pseudospectral computational framework for computing the propagation characteristics of optical 

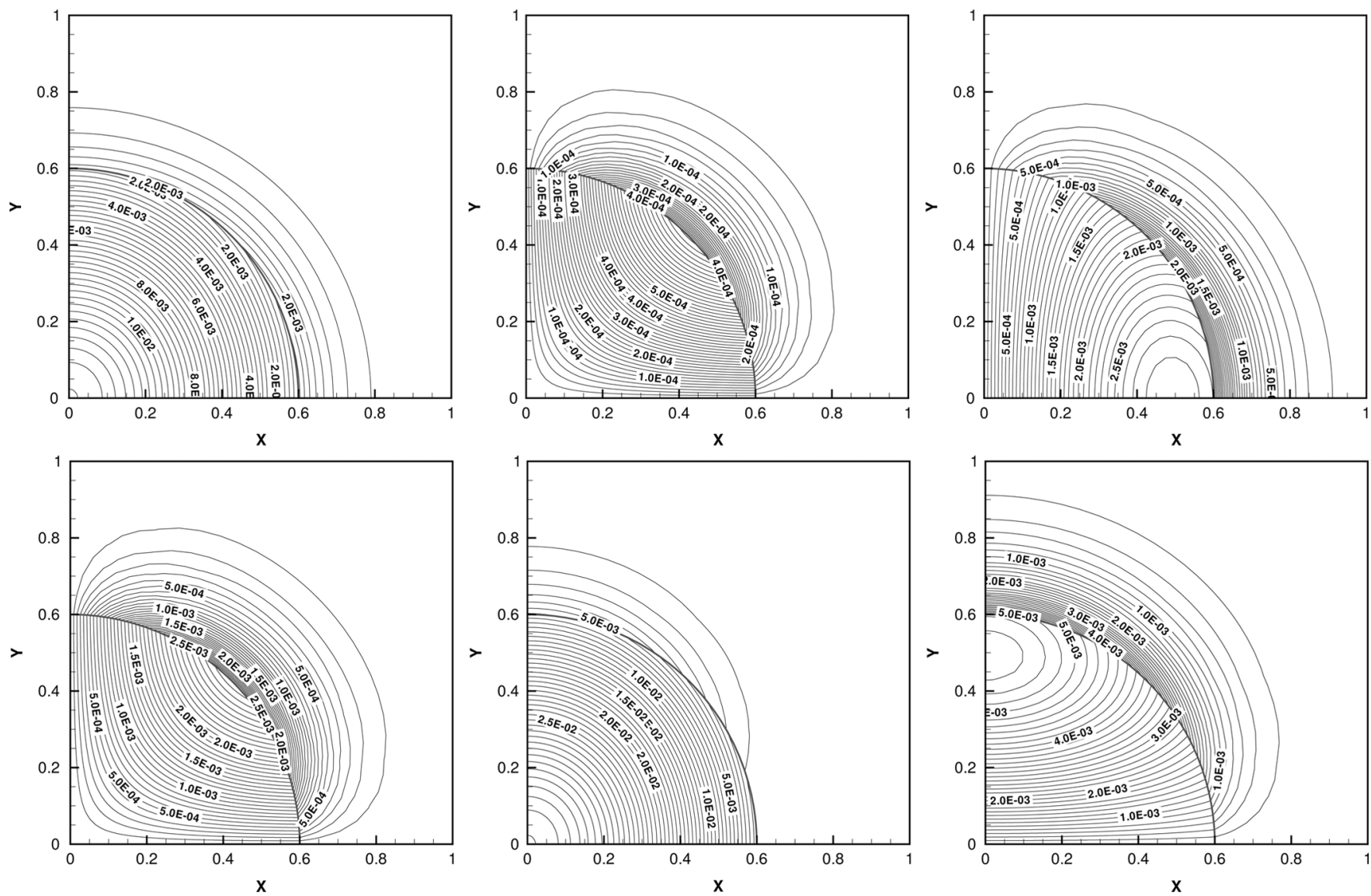

Fig. 6. Field amplitude contour line plots of the fiber waveguide problem. $\left|E_{x}\right|,\left|E_{y}\right|$, and $\left|E_{z}\right|$ are placed on the top row from left to right. $\left|H_{x}\right|,\left|H_{y}\right|$, and $\left|H_{z}\right|$ are placed on the bottom row from left to right.

TABLE III

CONVERGENCE STUdy OF THE Fiber WAVEGUIDE. $n_{\text {eff }}^{\prime}=$ 2.684019321609156, REFERENCED EFFECTIVE INDEX OF THE HE 11 Fundamental Mode at the Operating WaVElength $\lambda_{0}=1.5 \mu \mathrm{M}$

\begin{tabular}{||c|c|c||c|c|c|c|c||}
\hline$N$ & $\left|\delta n_{e f f}\right|$ & $\zeta$ & $N$ & $r_{1}$ & $\zeta$ & $r_{2}$ & $\zeta$ \\
\hline 4 & $7.98 \mathrm{E}-04$ & - & 8 & $3.11 \mathrm{E}-03$ & - & $8.52 \mathrm{E}-03$ & \\
6 & $1.54 \mathrm{E}-05$ & 9.7 & 12 & $1.87 \mathrm{E}-05$ & 12.6 & $5.07 \mathrm{E}-05$ & 12.6 \\
8 & $1.07 \mathrm{E}-07$ & 17.2 & 16 & $6.71 \mathrm{E}-08$ & 19.5 & $1.81 \mathrm{E}-07$ & 19.5 \\
10 & $4.25 \mathrm{E}-10$ & 24.8 & 20 & $2.34 \mathrm{E}-10$ & 25.3 & $6.28 \mathrm{E}-10$ & 25.3 \\
12 & $9.68 \mathrm{E}-12$ & 20.8 & 24 & $1.33 \mathrm{E}-12$ & 28.3 & $3.83 \mathrm{E}-12$ & 27.9 \\
\hline
\end{tabular}

wave fields in waveguides. The mathematical formulation is based on the Maxwell equations in frequency domain, and the required boundary operator to complete the formulation was identified. Relationships between the mathematically derived boundary operator and the common boundary conditions were also established. We constructed a multidomain Legendre pseudospectral scheme with boundary condition weakly imposed through the penalty methodology. Numerical experiments were conducted and we observed the expected spectral convergence of the scheme for both smooth problems and those having finite jump discontinuities at material interfaces. However, for problems involving singular wave fields occurring at sharp dielectric corners, the numerical experiments showed that the spectral convergence of the scheme is ruined and the order of the convergence is at most first-order accurate in the global sense. Nevertheless, the present pseudospectral method remains an efficient approach to compute the results compared to those obtained by finite difference methods [5].

For waveguide analysis, a common approach is solving fullvectorial wave equations in terms of the two transverse magnetic field components. The total number of discrete equations raised by the transverse field formulations is less than that raised by the present six-component formulation. We admit that the present method requires more computational work. To overcome this issue, we have reformulated our method to reduce the number of equations. The idea is mimicking the procedure of obtaining the full-vectorial wave equations from the Maxwell equations, which involves taking the partial derivatives of the first-order system of equations and then conducting algebraic eliminations to yield decoupled second-order wave equations. For the present method, we can write (16) and (17) into their continuous representations, and then take derivatives and conduct algebraic eliminations to obtain a system of second-order equations composed of the transverse magnetic field components and the nontransverse electric field component. Thus, the total number of discrete equations is reduced and the computational work can be reduced. To completely eliminate the nontransverse electric field requires further investigation. The details of this study will be further conducted and presented elsewhere in the future.

Although the present formulation results into a larger system compared to the transverse magnetic field components ap- 

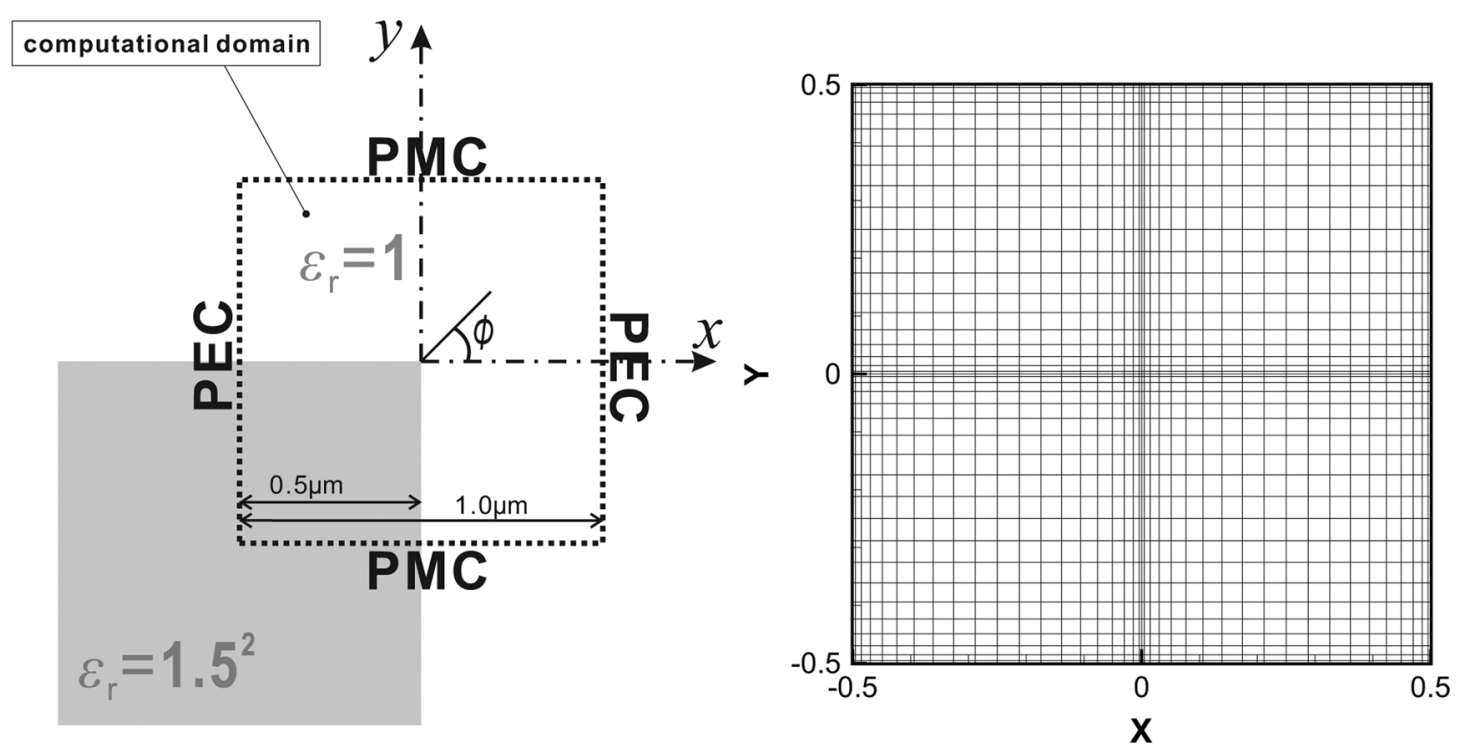

Fig. 7. Cross-sectional view of (left) a dielectric waveguide and (right) a computational mesh composed of four subdomains.

TABLE IV

Grid Convergence Study of the Channel Waveguide. $n_{\text {eff }}^{\prime}=1.27627404 \pm 10^{-8}$, Reference EFfective IndeX For the Fundamental Mode at the Operating WaVelength $\lambda_{0}=1.5 \mu \mathrm{M}$ Adopted From [5]

\begin{tabular}{||c|c|c||c|c|c|c|c|}
\hline$N$ & $n_{e f f}$ & $\left|\delta n_{e f f}\right|$ & $N$ & $r_{1}$ & $\zeta_{r_{1}}$ & $r_{2}$ & $\zeta_{r_{2}}$ \\
\hline 4 & 1.27627574310227 & $1.70 \mathrm{E}-06$ & 8 & $6.91 \mathrm{E}-03$ & - & $4.77 \mathrm{E}-04$ & \\
6 & 1.27627406000974 & $2.00 \mathrm{E}-08$ & 12 & $5.22 \mathrm{E}-03$ & 0.69 & $2.14 \mathrm{E}-04$ & 1.97 \\
8 & 1.27627401416807 & $2.58 \mathrm{E}-08$ & 16 & $4.25 \mathrm{E}-03$ & 0.71 & $1.19 \mathrm{E}-04$ & 2.02 \\
10 & 1.27627402128922 & $1.87 \mathrm{E}-08$ & 20 & $3.62 \mathrm{E}-03$ & 0.71 & $7.58 \mathrm{E}-05$ & 2.04 \\
12 & 1.27627402754483 & $1.24 \mathrm{E}-08$ & 24 & $3.17 \mathrm{E}-03$ & 0.72 & $5.20 \mathrm{E}-05$ & 2.06 \\
\hline
\end{tabular}

TABLE V

EFFective IndeX of THE Fundamental Mode of the ChanNel WaVEguide Computed by the SCheme With Very Dense Meshes With $\varepsilon_{r}=2.25$ OPERATING WAVELENGTH $\lambda_{0}=1.5 \mu \mathrm{M}$

\begin{tabular}{||c|c||}
\hline$N$ & $n_{e f f}$ \\
\hline 40 & 1.276274037658855 \\
44 & 1.276274037716481 \\
48 & 1.276274037755042 \\
52 & 1.276274037781696 \\
\hline
\end{tabular}

TABLE VI

EFFeCtive Index of the Fundamental Mode of the ChanNel WAVEGUide COMPUTED BY THE SCHEME WiTH Very DENSE MESHES WitH $\varepsilon_{r}=8$. OPERATING WAVELENGTH $\lambda_{0}=1.5 \mu \mathrm{M}$

\begin{tabular}{||c|c||}
\hline$N$ & $n_{e f f}$ \\
\hline 8 & 2.656796890564512 \\
16 & 2.656796916630881 \\
24 & 2.656796921885117 \\
32 & 2.656796923270166 \\
40 & 2.656796923780370 \\
\hline
\end{tabular}

proach, the present method may have a possible advantage on solving problems involving tensor permittivity. For this situation, the full-vectorial formulation may involve cross differentiation terms, which may become complicated for imposing boundary conditions. However, in the present method, tensor permittivity will only affect the matrix $A_{3}$ which is a low-order term in (4). Consequently, the present boundary formulation, which is related to the $A_{1}$ and $A_{2}$ matrices, may remain valid. Detail investigations will be explored in the future.

Before the end of this paper, we briefly discuss two issues related to the further development of the present method. The first one regards computing waves propagating toward to far fields along the transverse plane. In this study, we only conduct experiments either for wave problems defined on closed domain or for problems where the waves are evanescent in the far field, so the domain can be terminated into a finite region supported by artificial boundary conditions. Because of the lack of methods on absorbing waves propagating toward to far zone for the present formulation, the performance of the scheme on solving leaky mode solutions of certain optical waveguide structures has not yet been examined. Hence, to extend the applicability of the present method for calculating leaky modes of waveguides, it is worth to develop suitable perfectly matched layer methods to absorb outgoing waves and maintain the solution accuracy in the guiding regions. The second issue regards the blow-up behavior of wave fields at dielectric corners. Although the present formulation remains a competitive approach, compared to other finite difference methods, for computing the propagation characteristics of waveguides involving dielectric corners, the singular nature does ruin the exponential convergence order of the scheme. It would be interesting to develop methods to enhance the convergence order, so the computations can be more efficient and accurate to resolve the singular behavior of fields at dielectric corners. We hope to report this development in the future. 


\section{ACKNOWLEDGMENT}

The authors would like to thank the National Center for HighPerformance Computing, Hsinchu, Taiwan, for providing useful computing resources.

\section{REFERENCES}

[1] K. Bierwirth, N. Schulz, and F. Arndt, "Finite-difference analysis of rectangular dielectric waveguide structures," IEEE Trans. Microw. Theory Tech., vol. 34, no. 11, pp. 1104-1113, Jan. 1986.

[2] M. S. Stern, "Semivectorial polarized finite difference method for optical waveguides with arbitrary index profiles," IEE Proc. J. Optoelectron., vol. 135 , no. 1 , pp. 56-63, 1988.

[3] C. Vassallo, "Improvement of finite difference methods for step-index optical waveguides," IEE Proc. J. Optoelectron., vol. 139, no. 2, pp. 137-142, 1992.

[4] P. Lüsse, P. Stuwe, J. Schüle, and H.-G. Unger, "Analysis of vectorial mode fields in optical waveguides by a new finite difference method," J. Lightw. Technol., vol. 12, no. 3, pp. 487-494, Mar. 1994.

[5] G. R. Hadley, "High-accuracy finite-difference equations for dielectric waveguide analysis: II. Dielectric corners," J. Lightw. Technol., vol. 20, no. 7, pp. 1219-1231, Jul. 2002.

[6] N. Thomas, P. Sewell, and T. M. Benson, "A new full-vectorial higher order finite-difference scheme for the modal analysis of rectangular dielectric waveguides," J. Lightw. Technol., vol. 25, no. 9, pp. 2563-2570, Sep. 2007.

[7] Y. P. Chiou, Y. P. Chiang, and H. C. Chang, "Improved three-point formulas considering the interface conditions in the finite-difference analysis of step-index optical devices," J. Lightw. Technol., vol. 18, no. 2, pp. 243-251, Feb. 2000.

[8] S. Zhao, "Full-vectorial matched interface and boundary (MIB) method for the modal analysis of dielectric waveguides," J. Lightw. Technol., vol. 26, no. 14, pp. 2251-2259, Jul. 2008.

[9] S. Zhao, "High-order matched interface and boundary methods for the Helmholtz equation in media with arbitrarily curved interfaces," $J$ Comput. Phys., vol. 229, pp. 3155-3170, 2010.

[10] M. Koshiba and K. Inoue, "Simple and efficient finite-element analysis of microwave and optical waveguides," IEEE Trans. Microw. Theory Tech., vol. 40, no. 2, pp. 371-377, Feb. 1992.

[11] J. F. Lee, D. K. Sun, and Z. J. Cendes, "Tangential vector finite element for electromagnetic field computation," IEEE Trans. Magn., vol. 27, no. 5, pp. 4032-4035, Sep. 1991.

[12] M. Koshiba, S. Maruyama, and K. Hirayama, "A vector finite element method with the high-order mixed-interpolation-type triangular elements for optical waveguiding problems," J. Lightw. Technol., vol. 12, no. 3, pp. 495-502, Mar. 1994

[13] D. U. Li and H. C. Chang, "An efficient full-vectorial finite-element modal analysis of dielectric waveguides incorporating inhomogeneous elements across dielectric discontinuities," IEEE J. Quantum Electron., vol. 36, no. 11, pp. 1251-1261, Nov. 2000.

[14] P.-J. Chiang, C.-L. Wu, C.-H. Teng, C.-S. Yang, and H.-C. Chang, "Full-vectorial optical waveguide mode solvers using multidomain pseudospectral frequency-domain (PSFD) formulations," IEEE $J$. Quantum Electron., vol. 44, no. 1, pp. 56-66, Jan. 2008.

[15] C.-C. Huang, C.-C. Huang, and J.-Y. Yang, "An efficient method for computing optical waveguides with discontinuous refractive index profiles using spectral collocation method with domain decomposition," $J$. Lightw. Technol., vol. 21, no. 10, pp. 2284-2296, Oct. 2003.

[16] C.-C. Huang, C.-C. Huang, and J.-Y. Yang, "A full-vectorial pseudospectral modal analysis of dielectric optical waveguides with stepped refractive index profiles," IEEE J. Quantum Electron., vol. 11, no. 2, pp. 457-465, Mar./Apr. 2005.

[17] J. Xiao and X. Sun, "Full-vectorial mode solver for anisotropic optical waveguides using multidomain spectral collocation method," Opt. Commun., vol. 283, pp. 2835-2840, 2010.

[18] J. S. Hesthaven, S. Gottlieb, and D. Gottlieb, Spectral Methods for Time-Dependent Problems. Cambridge, U.K.: Cambridge Univ. Press, 2007.

[19] D. Funaro and D. Gottlieb, "A new method of imposing boundary conditions in pseudospectral approximations of hyperbolic equations," Math. Comput., vol. 51, pp. 599-613, 1988.

[20] C. H. Teng, B. Y. Lin, H. C. Chang, H. C. Hsu, C. N. Lin, and K. A Feng, "A Legendre pseudospectral penalty scheme for solving timedomain Maxwell's equations," J. Sci. Comput., vol. 36, pp. 351-390, 2008 .
[21] B. Y. Lin, H. C. Hsu, C. H. Teng, H. C. Chang, J. K. Wang, and Y. L. Wang, "Unraveling near-field origin of electromagnetic waves scattered from silver nanorod arrays using pseudo-spectral time-domain calculation," Opt. Exp., vol. 17, pp. 14211-14228, 2009.

[22] B. Y. Lin, C. H. Teng, H. C. Chang, H. H. Hsiao, J. K. Wang, and Y. L. Wang, "Pseudospectral modeling of nano-optics in Ag sphere arrays," J. Sci. Comput., vol. 45, pp. 429-446, 2010.

[23] S. F. Chiang, B. Y. Lin, C. H. Teng, and H. C. Chang, "Improved analysis of rectangular dielectric waveguides based on a Legendre pseudospectral penalty scheme," presented at the presented at the Integr. Photon. Res., Silicon and Nano Photon., Washington, DC, 2010, Paper IWH8.

[24] W. J. Gordon and C. A. Hall, "Transfinite element methods: Blendingfunction interpolation over arbitrary curved element domains," Numer. Math., vol. 21, pp. 109-129, 1973.

[25] R. Collin, Field Theory of Guided Waves. New York: McGraw-Hill, 1960.

[26] W. Lu and Y. Y. Lu, "Waveguide mode solver based on Neumann-toDirichlet operators and boundary integral equations," J. Comput. Phys., vol. 231, pp. 1360-1371, 2012.

Shun-Fan Chiang received the B.S. degree in mechanical engineering from National Taiwan University, Taipei, Taiwan, in 2006, and the M.S. degree in optoelectronics engineering from National Taiwan University, Taipei, in 2008.

Since 2008, he has been with Hon Hai Precision Industry Ltd., Taipei. His research interests include simulation programming design, control system design, and mechanical design.

Bang-Yan Lin received the B.S. degree in electronic engineering from Chung Yuan Christian University, Chung-Li, Taiwan, in 1997, and the M.S. and Ph.D. degrees in communication engineering from the National Taiwan University, Taipei, Taiwan, in 2002 and 2009, respectively.

He was involved in the development of the pseudospectral method and its applications to optoelectronics, including photonic crystals, nanophotonics, and plasmonics. In 2009, he joined Taiwan Semiconductor Manufacturing Company, Hsinchu, Taiwan, as a Principal Engineer, where he is currently involved in research on MOSFET modeling.

Hung-Chun Chang (S'78-M'83-SM'00) was born in Taipei, Taiwan, on February 8,1954 . He received the B.S. degree from National Taiwan University, Taipei, in 1976, and the M.S. and Ph.D. degrees from Stanford University, Stanford, CA, in 1980 and 1983, respectively, all in electrical engineering.

From 1978 to 1984, he was with the Space, Telecommunications, and Radioscience Laboratory, Stanford University. In August 1984, he joined the Department of Electrical Engineering (EE), National Taiwan University (NTU), where he is currently a Distinguished Professor. He was the NTU Himax Chair Professor in 2011. He served as the Vice Chairman of the EE Department from 1989 to 1991, and the Chairman of the newly established Graduate Institute of Electro-Optical Engineering, NTU, from 1992 to 1998. His current research interests include the electromagnetic theory, design, and application of photonic structures and devices for fiber optics, integrated optics, optoelectronics, nanophotonics, and plasmonics.

Dr. Chang served as the Institute of Electronics, Information and Communication Engineers, Japan, Overseas Area Representative in Taipei from 2002 to 2007. He is a Fellow of the Optical Society of America and the Electromagnetics Academy.

Chun-Hao Teng was born in Tainan, Taiwan, on February 14, 1970. He received the Diploma degree in mechanical engineering from the National Taipei Institute of Technology, Taipei, Taiwan, in 1990, the M.S. degree in mechanical engineering from Clarkson University, Potsdam, NY, in 1996, and the M.S. and $\mathrm{Ph}$.D. degrees in applied mathematics from Brown University, Providence, RI, in 1998 and 2001, respectively.

From 2003 to 2009, he was an Assistant Professor in the Department of Mathematics, National Cheng Kung University, Tainan. He is currently an Assistant Research Fellow in the Department of Applied Mathematics and the Center of Mathematical Modeling and Scientific Computing, National Chiao Tung University, Hsinchu, Taiwan. His research interests include the developments and applications of high-order numerical methods for partial differential equations. 
Chih-Yu Wang received the B.S. degree from the Department of Engineering Science, National Cheng Kung University, Tainan, Taiwan, in 2004, and the M.S. degree from the Graduate Institute of Electronics Engineering, National Taiwan University, Taipei, Taiwan, in 2006, where she is currently working toward the Ph.D. degree.

Her current research interests include the pseudospectral electromagnetics modeling in frequency domain.
Shih-Yung Chung received the B.S. degree from the Department of Electrical Engineering, National Cheng Kung University, Tainan, Taiwan, in 2004, and the M.S. degree from the Graduate Institute of Electronics Engineering, National Taiwan University, Taipei, Taiwan, in 2006, where he is currently working toward the Ph.D. degree.

His current research interests include the electromagnetic simulations using the pseudospectral time domain method. 